\title{
The denatured state of HIV-1 protease under native conditions
}

\author{
Heike Rösner ${ }^{1}$, Martina Caldarini ${ }^{2}$, Gregory Potel $^{3}$, Daniel Malmodin ${ }^{1}$, Maria Vanoni ${ }^{4}$, \\ Alessandro Aliverti ${ }^{5}$, Ricardo Broglia ${ }^{4}$, Birthe Kragelund ${ }^{1}$, and Guido Tiana ${ }^{4}$ \\ ${ }^{1}$ University of Copenhagen \\ ${ }^{2}$ University of Milano \\ ${ }^{3}$ Lawrence Livermore National Laboratory \\ ${ }^{4}$ University of Milan \\ ${ }^{5}$ Universita degli Studi di Milano
}

June 18, 2021

\begin{abstract}
The denatured state of several proteins has been shown to display transient structures that are relevant for folding, stability and aggregation. To detect them by nuclear magnetic resonance (NMR) spectroscopy, the denatured state must be stabilized by chemical agents or changes in temperature. This makes the environment different from that experienced in biologically relevant processes. Using high-resolution heteronuclear NMR spectroscopy, we have characterized several denatured states of a monomeric variant of HIV-1 protease induced by different concentrations of urea, guanidinium chloride and acetic acid. We have extrapolated the chemical shifts and the relaxation parameters to the denaturant-free denatured state at native conditions, showing that they converge to the same values. Subsequently, we characterized the conformational properties of this biologically relevant denatured state under native conditions by advanced molecular dynamics simulations and validated the results by comparison to experimental data. We show that the denatured state of HIV-1 protease under native conditions displays rich patterns of transient native and non-native structures, which could be of relevance to its guidance through a complex folding process.
\end{abstract}

\section{Introduction}

The denatured state $D_{0}$ that proteins populate transiently under native conditions ${ }^{1}$ is important to determine their folding ${ }^{2}$, stability ${ }^{3}$, aggregation ${ }^{4}$ and misfolding ${ }^{5}$, properties that can have direct implication for disease states. Except for a few specific proteins ${ }^{6-8}, D_{0}$ is so poorly populated that it escapes experimental observation. To overcome this problem, induced denatured states can be stabilized by chemical agents like urea, guanidine hydrochloride $(\mathrm{GdmCl})$ or acids, populating the states $D_{\text {urea }}, D_{G d m C l}$ and $D_{\text {acid }}$, respectively; states that are not necessarily similar to $D_{0}$ and which show variation amongst themselves. However, from a thermodynamic point of view, calorimetry experiments ${ }^{9}$ showed that the unfolding enthalpy of lysozyme, denatured by $\mathrm{pH}, \mathrm{GdmCl}$ and temperature is identical once the energy associated with the denaturant mean (e.g., the ionization energy in the case of $\mathrm{pH}$ ) was subtracted. From these data, it was concluded that the states denatured by different means are thermodynamically indistinguishable ${ }^{9}$.

One could then ask whether the conformational properties of the different denatured states $D_{\text {urea }}, D_{G d m C l}$, $D_{a c i d}$ and $D_{0}$ are similar as well. Although these states were originally believed to be randomly disordered ${ }^{10}$, recent studies have revealed them to contain transient secondary ${ }^{11-15}$ and even tertiary structures ${ }^{16,17}$. Such results were made possible mainly thanks to the development of NMR techniques and in particular of secondary chemical shift analysis.

In the present work, we studied the denatured states of a monomeric variant of human immunodeficiency 
virus (HIV)-1 protease11This is exactly the same protein used in ref. 27, in spite of the unfortunate notation used in that reference. (mHIV-1-PR ${ }_{1-95}$ ), a protein necessary for HIV-1 to replicate in infected cells ${ }^{18}$. The denatured state of HIV-1 protease under native conditions is particularly important because it was suggested as a possible target of antiretroviral drugs that prevent the correct folding of the protein and thus of its enzymatic activity ${ }^{19-21}$. Moreover, the native conformation of mHIV-1-PR ${ }_{1-95}$ displays a topology, which is more complex than that of typical proteins of comparable size, a feature possibly encoded also in its denatured state. In fact, its native conformation displays two pseudo-knots and the associate Plaxco's contact order $^{22}$, quantifying the non-locality of native contacts, is 15, much larger than the values 8-10 of typical proteins of comparable length.

HIV-1 protease is an aspartic acid protease, which in its active form exists as a homodimer ${ }^{23}$ (Fig 1a). Analysis of its folding kinetics identified a monomeric intermediate that associates to form the native dimer structure $^{24}$. Deletion of the last four C-terminal residues stabilizes a monomeric, folded form ${ }^{25}$. The native structure of this mHIV-1-PR $1-95$, predominantly contains $\beta$-sheet structure and a C-terminal $\alpha$-helix ${ }^{18}$, highly similar to the structure in the dimer (cf. Fig 1b). Both the unfolding and refolding kinetics of mHIV-1PR studied in urea by fluorescence display two time scales, suggesting the presence of at least one kinetic intermediate and the typical refolding time of mHIV-1-PR $1-95$ is of the order of a minute ${ }^{24}$. Also, mechanical unfolding experiments suggest the presence of folding and unfolding intermediates ${ }^{26}$. Interestingly, mHIV1-PR was shown to display cold denaturation well above zero degrees Celsius ${ }^{27}$, a feature that allowed us to compare the denatured states $D_{\text {urea }}, D_{G d m C l}$ and $D_{\text {acid }}$ to a further state $D_{\text {cold }}$.

The native and non-native states of the wild-type and of several variants of HIV-1-PR were also characterized both in silico and in vivo ${ }^{28-30}$. In spite of its central role as a target for anti-retroviral therapies, biochemical and biophysical data on HIV-1 protease are still limited. A tethered dimer in $\mathrm{GdmCl}^{31,32}$, a wild-type dimer in acetic acid ${ }^{33}$ and HIV-1-protease embedded in its viral precursor protein in urea ${ }^{34}$ constitute some of these states. However, none of these studies were performed on the same variant of the protein, prohibiting a direct comparison of the results.

\section{Hosted file}

image1.emf available at https://authorea.com/users/420414/articles/526767-the-denaturedstate-of-hiv-1-protease-under-native-conditions

Figure 1: The native conformation of HIV-1-PR and mHIV-1-PR $1-95$. a) Structure of the HIV-1-PR homodimer (PDB code 1BVG). The active site is highlighted by yellow spheres. b) Structure of the 1-95 variant mHIV-1-PR $1-95$ (PDB code 1Q9P). c) The sequence of mHIV-1-PR; the active site is highlighted in yellow.

In the present work we have performed titration experiments in different chemical denaturants using the exact same mHIV-1-PR $1-95$ variant. This allowed us to monitor how the conformational properties of the denatured state depend on the kind and concentration of the denaturing agent, eventually extrapolating the properties of $D_{0}$. The main quantity we investigated was the secondary chemical shifts, measured by heteronuclear NMR experiments. A non-trivial problem one has to face is then to interpret these data in terms of conformational properties of the protein. To assist us in this goal, we performed advanced molecular

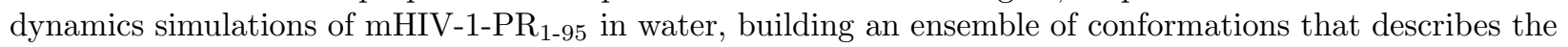
denatured $\operatorname{state} D_{0}$. The correctness of the $\operatorname{simulated} D_{0}$ was validated by back-calculating the secondary chemical shifts from the simulation and comparing them with those obtained from the extrapolation to zero denaturant of the NMR results.

\section{Materials and Methods}

Protein expression, purification and sample preparation. A synthetic gene encoding the HIV-1

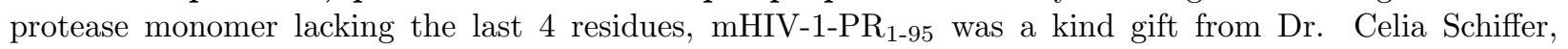
University of Massachusetts Medical School, and was cloned into a pET11a vector. The protein was expressed in Escherichia coli Rosetta (DE3) cells upon induction with $0.2 \mathrm{mM}$ IPTG. For the synthesis of isotope labeled protein, Spectra9 LB media (Euriso-top, France - cod. CGM-3030-CN-1, 1L) enriched with ${ }^{15} \mathrm{~N}$ and 
${ }^{13} \mathrm{C}$ was used. Cells obtained from $0.4 \mathrm{~L}$ of culture were lysed by sonication at $4^{\mathrm{O}} \mathrm{C}$ in extraction buffer: $20 \mathrm{mM}$ Tris/HCl, $1 \mathrm{mM}$ EDTA and $10 \mathrm{mM}$ DTT, $\mathrm{pH}$ 8. The protein was refolded as described previously ${ }^{26}$. For spectroscopic measurements, the protein was dialyzed against $20 \mathrm{mM}$ sodium phosphate, $\mathrm{pH} 6.0$.

Fluorescence and CD experiments. Fluorescence experiments were performed with a Varian Eclipse fluorimeter on $4 \mu \mathrm{M}$ protein in $20 \mathrm{mM}$ sodium phosphate at $\mathrm{pH} 6.0$ and $25^{\circ} \mathrm{C}$ by adding different concentrations of denaturant. CD measurements were conducted at $230 \mathrm{~nm}$ and a protein concentration of $15 \mu \mathrm{M}$ in $20 \mathrm{mM}$ sodium phosphate, $\mathrm{pH} 6$, and containing different amounts of denaturant at $25^{\circ} \mathrm{C}$ using a JASCO J810 spectropolarimeter and a $1 \mathrm{~mm}$ path length. A total of 120 data points were recorded over 1 minute and averaged. The actual urea and $\mathrm{GdmCl}$ concentrations were confirmed by refractive index measurements. For the temperature transition, CD measurements were conducted at $205 \mathrm{~nm}$ and a protein concentration of $10 \mu \mathrm{M}$ in $20 \mathrm{mM}$ sodium phosphate, $\mathrm{pH}$. The temperature was increased in $1{ }^{\circ} \mathrm{C}$ steps from 3 to $20{ }^{\circ} \mathrm{C}$ and in $2{ }^{\circ} \mathrm{C}$ steps from 20 to $90{ }^{\circ} \mathrm{C}$ using a Peltier control unit. To account for the slow refolding kinetics, each point was allowed to equilibrate 5 minutes prior to detection.

NMR experiments. Backbone assignment and $R_{1}, R_{2}$ and hetNOE relaxation experiments . All NMR spectra were recorded either on an Agilent DD2 $800 \mathrm{MHz}$ or a Varian INOVA $750 \mathrm{MHz}$ spectrometer using a room temperature probe, and standard pulse programs from the Vnmrj BioPack. For assignment, we prepared 11 different aliquots of ${ }^{15} \mathrm{~N}-{ }^{13} \mathrm{C}$-labelled $\sim 200 \mu \mathrm{M}$ protein solution in $20 \mathrm{mM}$ sodium phosphate, $\mathrm{pH}$ 6.0, and 10 $\% \mathrm{D}_{2} \mathrm{O}(\mathrm{v} / \mathrm{v}), 125 \mu \mathrm{M}$ DSS (2,2-dimethyl-2-silanepentane-5-sulfonic acid) containing 4, 6 and $8 \mathrm{M}$ urea, 0.75, 1, 2 and $4 \mathrm{M} \mathrm{GdmCl}$, or $9 \%(\mathrm{v} / \mathrm{v}), 25 \%(\mathrm{v} / \mathrm{v})$, and $45 \%(\mathrm{v} / \mathrm{v})$ acetic acid, respectively, and one containing no extra additives. For relaxation experiments, identical samples were prepared containing a ${ }^{15} \mathrm{~N}$-labelled $\sim 200$ $\mu \mathrm{M}$ protein solution. The backbone nuclei were assigned using $\mathrm{HSQC}^{35}, \mathrm{HNCA} \mathrm{HNCO}^{36}, \mathrm{HN}(\mathrm{CA}) \mathrm{CO}^{37}$, $\mathrm{HNCOCA}^{38}, \mathrm{HNCACB}^{39}, \mathrm{CBCACONH}^{40}, \mathrm{HNN}^{41}$ and ${ }^{15} \mathrm{~N}$-edited NOESY-HSQC ${ }^{42}$ spectra recorded at $25^{\circ} \mathrm{C}$ for the samples containing 4 and $8 \mathrm{M}$ urea, and using only HSQC, HNCA, HNCO, HNCOCA, HNN and ${ }^{15} \mathrm{~N}-$ edited NOESY-HSQC spectra for $1 \mathrm{M} \mathrm{GdmCl}$ and $25 \%$ (v/v) acetic acid. For the remaining samples, only the HSQC, HNCA, HNCOCA and HNCO spectra were used for backbone assignment. The assignment was completed for $95 \%$ of all non-proline residues for samples containing acetic acid, $96 \%$ for samples containing $\mathrm{GdmCl}, 97 \%$ for samples containing urea and $97 \%$ for cold denatured protein.

To analyze the $T_{1}$ and $T_{2}$ relaxation times and heteronuclear NOEs (hetNOEs), five series of spectra were recorded on ${ }^{15} \mathrm{~N}$-labelled protein in $20 \mathrm{mM}$ sodium phosphate, $\mathrm{pH} 6.0$, and $10 \% \mathrm{D}_{2} \mathrm{O}(\mathrm{v} / \mathrm{v}), 125 \mu \mathrm{M}$ DSS, also containing 4 or $8 \mathrm{M}$ urea, $1 \mathrm{M} \mathrm{GdmCl}$ or $25 \%(\mathrm{v} / \mathrm{v})$ acetic acid, at $25{ }^{\circ} \mathrm{C}^{43}$. We chose 8 different delay times: $0 \mathrm{~ms}, 100 \mathrm{~ms}, 200 \mathrm{~ms}, 300 \mathrm{~ms}, 500 \mathrm{~ms}, 700 \mathrm{~ms}, 900 \mathrm{~ms}$ and $1200 \mathrm{~ms}$ for recording $\mathrm{T}_{1}$ and 9 different delay times: $10 \mathrm{~ms}, 50 \mathrm{~ms}, 90 \mathrm{~ms}, 130 \mathrm{~ms}, 170 \mathrm{~ms}, 190 \mathrm{~ms}, 210 \mathrm{~ms}, 230 \mathrm{~ms}$ and $250 \mathrm{~ms}$ for recording $\mathrm{T}_{2}$ relaxation times. For the hetNOE a relaxation delay of $8 \mathrm{~s}$ was used.

PFG NMR diffusion experiments. The above described protein samples were used to record sets of 60 bipolar pulse-pair stimulated echo experiments using a watergate scheme for water suppression with varying gradient strength ${ }^{44}$. As internal reference, $0.5 \%(\mathrm{v} / \mathrm{v})$ dioxane was added to all samples to correct for viscosity effects by the solvent. All spectra were obtained at $25^{\circ} \mathrm{C}$ using 32 transients on a $750 \mathrm{MHz}$ Varian INOVA spectrometer.

2-D and 3-D NMR spectra processing. The X-carrier frequency was determined by referencing to internal DSS. The DSS frequency was obtained from a $1 \mathrm{D}{ }^{1} \mathrm{H}$ spectra recorded immediately before the remaining experiments. Indirect referencing was used in the ${ }^{15} \mathrm{~N}$ and ${ }^{13} \mathrm{C}$ dimensions by use of conversion factors ${ }^{45}$. The spectra were processed using nmrPipe ${ }^{46}$ and qMDD ${ }^{47}$. Spectrometer frequencies and carrier frequencies in ppm were inserted with 4 decimals. Zero-filling to nearest power of 2 was used. The processed spectra were assigned and analyzed in CcpNmr Analysis ${ }^{48}$. The assigned HSQC spectra were further used to extract the relaxation decays from the series of spectra recorded to determine the $\mathrm{T} 1$ and $\mathrm{T} 2$ relaxation times. Relaxation decay curves were fitted to single exponentials and relaxation times determined using therelax software ${ }^{49,50}$ The values of $R_{1}, R_{2}$ and the hetNOE recorded at 17.6 Tesla were used to derive the spectral density function at three frequencies $\left(0, \omega_{\mathrm{H}}\right.$ and $\left.\omega_{\mathrm{N}}\right)$ analyzed by reduced spectral density mapping using $\operatorname{relax}^{49,50}$. 
DOSY processing . Each set of $601 \mathrm{D}^{1}{ }^{1} \mathrm{H}$ spectra was separately processed and analyzed using The DOSY Toolbox $^{51}$ and MATLAB ${ }^{52}$. Spectra were phased in zero order and smoothed using a $10 \mathrm{~Hz}$ Lorentzian efficiently removing most visible noise. The function msbackadj was used rather than the internal DOSY Toolbox baseline correction routine.

Analysis of the chemical shifts. Secondary chemical shifts associated with different atoms were systematized using the formula $\left(\Delta\left(\delta \mathrm{C}^{\alpha}\right)+\Delta\left(\delta \mathrm{C}^{\prime}\right)-0.5^{*} \Delta(\delta \mathrm{N})\right)^{7}$.

Fit of dynamics parameters. The R2 parameters were fitted with the function described in Eq. 3 in the Supplementary Materials of ref. ${ }^{53}$. The fit was done with a nonlinear least-square algorithm employing a Levenberg-Marquardt algorithm. To avoid overfitting, we performed fits with different number of exponentials, eventually choosing the minimum number of exponentials which gave a chi ${ }^{2}$ lower than 5 .

NMR data have been deposited at the BioMagResBank with the accession number: 25255.

Molecular dynamics simulations. The mHIV-1-PR $1-95$ system was described with the Amber 99SBdisp force field ${ }^{54}$ in Tip4/pd water and simulated with Gromacs $2020.4^{55}$. The protein was prepared in a dodecahedric box of $571 \mathrm{~nm}^{3}$ with 19160 water molecules and $4 \mathrm{Cl}^{-}$ions to neutralize the charge. A preliminary simulation of $50 \mathrm{~ns}$ at $700 \mathrm{~K}$ and constant volume was carried out, followed by $100 \mathrm{~ns}$ at $300 \mathrm{~K}$ and $1 \mathrm{~atm}$. From the latter simulation, 110 conformations were extracted to act as starting conformations of the production run. A replica-exchange simulation was then performed with 110 replicas whose temperature range from $300 \mathrm{~K}$ to $500 \mathrm{~K}$ for a total of $68 \mu \mathrm{s}$.

Once the first $30 \mathrm{~ns}$ were removed, the replica at $300 \mathrm{~K}$ was analyzed to validate the simulation against the NMR data. Secondary chemical shifts were calculated for each conformation with Sparta $+{ }^{56}$ and averaged over all of them. To calculate secondary chemical shifts, we used Bax's reference value ${ }^{56}$.

To predict the $\mathrm{R} 1$ relaxation parameters qualitatively we extracted 50 conformations from the $300 \mathrm{~K}$ trajectory, using each of them as starting point of a 1ns simulation at fixed temperature. The root mean square fluctuations around each of the 50 average conformations were calculated and then averaged together. The experimental R2 values were compared to the solvent-accessible surface area of each residue, averaged over the full $300 \mathrm{~K}$ trajectory.

The clustering of the $300 \mathrm{~K}$ trajectory was performed with a tailor-made Python code that uses the fraction $q$ of common contacts as underlying metric, normalized to the maximum between the numbers of contacts of the two structures. A contact is defined if the center of mass of two residues are closer than $0.65 \mathrm{~nm}$. In the calculation of $q$, only pairs of residues which were further apart by at least 3 other residues along the chain were considered.

\section{Results}

\section{Denaturation of mHIV-1}

Following the far-UV CD spectra of folded mHIV-1-PR $1-95$ and cold-denatured mHIV-1-PR $1-95$ (Fig. S1 in the SI), we observed nearly identical spectra over a wide range of wavelength spanning from 208-250 $\mathrm{nm}$. This is due to the presence of dominating aromatic contributions in the far-UV region ${ }^{57}$, which result in an atypical CD spectrum of a $\beta$-sheet protein. To monitor the unfolding temperature of $\mathrm{mHIV}-1-\mathrm{PR}_{1-95}$, we therefore chose to record the mean residue ellipticity at $205 \mathrm{~nm}$ as a function of increasing temperature from $3{ }^{\circ} \mathrm{C}$ to $90{ }^{\circ} \mathrm{C}$ (Fig. S2).

Besides cold denaturation occurring at $10{ }^{\circ} \mathrm{C}$, already described in ref. ${ }^{27}$, we observed heat denaturation with an apparent midpoint temperature, $T_{m}{ }^{a p p}$ of approximately $50{ }^{\circ} \mathrm{C}$ and a third transition at $80 \operatorname{degC}$, corresponding to the irreversible aggregation of the protein. Due to aggregation, the heat-denatured state was not considered for high resolution NMR studies. Under all conditions explored, the native state was never fully populated and hence all equilibrium unfolding transitions could not be satisfactorily fitted to a standard equilibrium transition curve. 
In the presence of increasing amounts of urea, mHIV-1-PR $\mathrm{PR}_{1-95}$ showed a very broad transition indicative of a non-cooperative unfolding (Fig. 2). Interestingly, close to $2 \mathrm{M}$ urea, the unfolding transition was more than $95 \%$ complete as judged from CD measurements, but not according to fluorescence emission. Thus, the data did not seem to agree with the expected behavior of a two-state unfolding mechanism. At

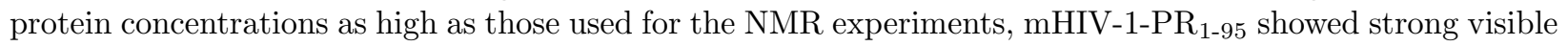
aggregation making reliable measurements below $4 \mathrm{M}$ urea impossible. In all NMR experiments, the protein was $>95 \%$ unfolded as judged from the CD signal. Monitoring the hydrodynamic radius $R_{h}$ by oulsed-filedgradient (PFG) NMR experiments at $4 \mathrm{M}$ urea showed that the hydrodynamic radius, $R_{h}=27.2+-0.5 \mathrm{~A}$, was comparable to data in ref. ${ }^{58}$. However, when increasing the urea concentration from 4 to $8 \mathrm{M}$ urea,

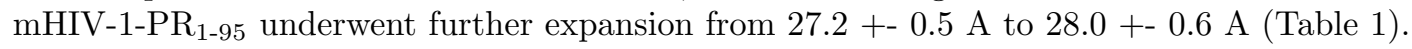

Compared to urea denaturation, the equilibrium transition curve was steeper and appeared more cooperative using GdmCl. The secondary structure of $\mathrm{mHIV}-1-\mathrm{PR}_{1-95}$ had already fully disappeared in the presence of less than $0.5 \mathrm{M} \mathrm{GdmCl}$ as monitored by $\mathrm{CD}$ (Fig. 2). Again, fluorescence emission indicated mHIV-1$\mathrm{PR}_{1-95}$ to be $>95 \%$ unfolded at a much higher concentration of denaturant than for CD, indicating that the monomer did not follow a two-state unfolding mechanism. At a denaturant concentration below $0.75 \mathrm{M}$ $\mathrm{GdmCl}$, protein aggregation was observed and NMR experiments were only recorded when more than $95 \%$ of the protein was denatured. Similar to the case in urea, the $R_{h}$ increased with increasing concentration of GdmCl. For three selected samples, the $R_{h}$ increased from $24+-0.5 \mathrm{~A}$ at $0.75 \mathrm{M} \mathrm{GdmCl}$ to $26.2+-0.5 \mathrm{~A}$ at $2 \mathrm{M} \mathrm{GdmCl}$ (Table 1).

The acid denatured state appears crucial for successful refolding of the dimeric protein ${ }^{59}$ and changes in protonation states can result in small but distinct differences in the preferences for local structure. The addition of just $0.1 \%$ acetic acid to $20 \mathrm{mM}$ sodium phosphate, $\mathrm{pH}$, caused the $\mathrm{pH}$ of the sample to drop to 4 , and in an identical buffer containing $0.75 \%$ acetic acid, $\mathrm{pH}$ was 3.4 . Addition of $5 \%$ acetic acid or more decreased $\mathrm{pH}$ below 3 , where dimeric HIV-1-PR is reported to be largely unfolded ${ }^{59}$. We observed a midpoint of denaturation at about $0.5 \%$ acetic acid, which corresponded to a measured $\mathrm{pH}$ of 3.6. From CD experiments, further addition of acetic acid caused additional structural changes even when full acid denaturation was complete, when judged from fluorescence emission spectra (Fig. 2). In addition, we observed an increase of $R_{h}$ from $27.2 \mathrm{~A}+-0.5$ at $9 \%$ acetic acid to $29.8+-0.6 \mathrm{~A}$ at $25 \%$ acetic acid (Table 1 ). This increase was significantly larger than for the other two denaturants.

Interestingly, in the absence of denaturant, $\mathrm{mHIV}-1-\mathrm{PR}_{1-95}$ is folded except for the N-terminal region ${ }^{18}$. In addition, the wild-type protein folds through a monomeric phase before dimerization ${ }^{24,30,60,61}$. Inspection of the HSQC spectrum of mHIV-1- $\mathrm{PR}_{1-95}$ recorded in $20 \mathrm{mM}$ sodium phosphate $(\mathrm{pH} 6.0)$ at 25 degC revealed a small but non-disputable second population. Under these experimental conditions, the folding rate of the monomer ${ }^{24}$ is about $1 \mathrm{~min}^{-1}$, the equilibrium thus being in the slower regime of chemical exchange for NMR experiments. Hence, the second set of peaks most likely originated from the denatured state $D_{0}$.

\section{Hosted file}

image2.emf available at https://authorea.com/users/420414/articles/526767-the-denaturedstate-of-hiv-1-protease-under-native-conditions

Figure 2: Equilibrium unfolding of HIV-1-PR $1-95$. Top: mean residue ellipticity at $230 \mathrm{~nm}$ of a $15 \mu \mathrm{M}$ HIV1- $\mathrm{PR}_{1-95}$ in $20 \mathrm{mM}$ sodium phosphate, $\mathrm{pH} 6$, measured in the presence of increasing concentrations of urea (left), $\mathrm{GdmCl}$ (middle) and acetic acid (right) at $25{ }^{\circ} \mathrm{C}$. Bottom: wavelength of maximum fluorescence emission of $4 \mu \mathrm{M}$ mHIV-1-PR $\mathrm{PR}_{1-95}$ sample $20 \mathrm{mM}$ sodium phosphate buffer, $\mathrm{pH} 6$, measured in the presence of increasing concentrations of urea, $\mathrm{GdmCl}$ or acetic acid, at $25{ }^{\circ} \mathrm{C}$ (excitation wavelength: $295 \mathrm{~nm}$ ).

\begin{tabular}{llllll}
\hline Urea & Urea & GdmCl & GdmCl & Acetic acid & Acetic acid \\
c(urea) & $R_{h}(\AA)$ & $\mathbf{c}(\mathbf{G d m C l})$ & $R_{h}(\AA)$ & $\%$ acetic acid & $R_{h}(\AA)$ \\
$4 \mathrm{M}$ & 27.2 & $0.75 \mathrm{M}$ & 24.0 & $9 \%$ & 27.2 \\
$6 \mathrm{M}$ & 27.7 & $1 \mathrm{M}$ & 25.8 & $25 \%$ & 29.8
\end{tabular}


All measurements were performed in $90 \% \mathrm{H}_{2} \mathrm{O} / 10 \% \mathrm{D}_{2} \mathrm{O}$ at $25{ }^{\circ} \mathrm{C}$. The experimental uncertainty was estimated to $\pm 2 \%$ based on triplicate measurements.

a The fitted data to this measurement were not available due to overlap with signals arising from acetic acid

Table 1: $R_{h}$ of mHIV-1-PR $1-95$ measured in different denaturants by PFG-NMR.

\section{Chemical shift analysis}

For each type of denaturant, the heteronuclear backbone resonances were assigned at three different denaturant concentrations (Fig. S3 in the SI). Moreover, the cold denatured state described in ref. ${ }^{27}$ was taken into account. The secondary chemical shifts of the protein in different denaturation conditions were rather similar to each other (Fig. S4).

To describe the transient structure in the denatured state under non-denaturing conditions, $\mathrm{C}^{\alpha}, \mathrm{C}^{\prime},{ }^{15} \mathrm{~N}$ and $\mathrm{H}^{\mathrm{N}}$ chemical shifts from individual titration series were extrapolated to the low intensity peaks observed at zero denaturant, as described in Fig. 3. As a result, in 16 cases the weak cross peaks observed at the position defined by the extrapolated values could be unambiguously assigned in the set of spectra recorded at physiological conditions at $25{ }^{\circ} \mathrm{C}$ in the absence of any denaturant. For these 16 cases, the assignment by extrapolation was cross-checked and confirmed by 3D backbone spectra. The same extrapolation procedure was applied to all residues and the remaining plots are shown in supplementary Fig. S3 together with 9 of the identified cross peaks in the HSQCs.

In Fig. 4 we report the extrapolated secondary chemical shifts for the $\mathrm{C}^{\alpha}, \mathrm{C}^{\prime}, \mathrm{N}$ and $\mathrm{H}^{\mathrm{N}}$ backbone atoms averaged over the chemical shifts obtained from the four different extrapolations under different denaturing conditions. In these plots, we make use of intrinsic reference (i.e., the chemical shifts at highest denaturant concentration), although other choices gave similar results (see Fig. S5 in the SI). The error bars indicate the associated standard error and quantify the precision of the assignment under native conditions. Most residues displayed small errors compared to the average. A few discrepancies were observed for charged residues. The largest deviations were associated with the titration with acetic acid and were observed for three aspartic acids, D29, D30 and D60, and the single histidine, H69. Weaker effects were seen for four glutamates, E21, E34, E35 and E65. All the fits are displayed in Fig. S6 in the SI

\section{Hosted file}

image3.emf available at https://authorea.com/users/420414/articles/526767-the-denaturedstate-of-hiv-1-protease-under-native-conditions

Figure 3: Convergence to the denatured state $\boldsymbol{D}_{0}$. a) ${ }^{15} \mathrm{~N}-\mathrm{HSQC}$ spectra (zoom) of mHIV-1-PR $1-95$ showing T80 in 12 different ${ }^{15} \mathrm{~N}-\mathrm{HSQC}$ spectra recorded under various denaturing conditions. All peaks converge to the same position for T80 in $D_{0}$. Red peaks: urea $8 \mathrm{M}$ (light red), urea $6 \mathrm{M}$ (medium red) and urea $4 \mathrm{M}$ (dark red). Green peaks: acetic acid 45\% (light green), acetic acid 25\% (medium green) and acetic acid 9\% (dark green). Blue peaks: GdmCl $4 \mathrm{M}$ (light blue), GdmCl $2 \mathrm{M}$ (medium blue), GdmCl $1 \mathrm{M}$ (dark blue). Purple peaks: sodium phosphate $20 \mathrm{mM}, \mathrm{pH}$ ) at $5^{\circ} \mathrm{C}$ (light violet), $15^{\circ} \mathrm{C}$ (dark violet). Black peaks: sodium phosphate $20 \mathrm{mM}, \mathrm{pH} 6$ at $25^{\circ} \mathrm{C}$ (denatured state $D_{0}$ ). b) Schematic of $\mathrm{T} 80{ }^{1} \mathrm{H}$ chemical shifts of mHIV-1-PR as a function of denaturant concentrations; the x-values are annotated in the graph (red: urea; blue: $\mathrm{GdmCl}$; green: acetic acid; purple: different temperatures; black: physiological condition at $25{ }^{\circ} \mathrm{C}\left(D^{\text {phys }}\right.$ state $\left.)\right)$. All ${ }^{1} \mathrm{H}$ chemical shifts converge to the $D_{0}$ state value. c) Same for ${ }^{15} \mathrm{~N}$ chemical shifts.

\section{Hosted file}

image4.emf available at https://authorea.com/users/420414/articles/526767-the-denaturedstate-of-hiv-1-protease-under-native-conditions 
Figure 4: Secondary chemical shift analysis extrapolated for the $D_{0}$ state of $\mathrm{mHIV}-1-\mathrm{PR} 1-95$. For each residue, the chemical shifts under different experimental conditions were extrapolated to zero denaturant. Here we report the average of these extrapolations. The secondary chemical shifts were calculated by the use of the intrinsic random coil reference. The error bars indicate the total error of the procedure. Different nuclei were monitored (a) $\mathrm{C}^{\alpha}$, (b) $\mathrm{C}^{\prime}$, (c) $\mathrm{N}$ and (d) $\mathrm{H}^{\mathrm{N}}$. The secondary structure of mHIV-1-PR $1-95$ is shown at the top.

\section{Polypeptide chain dynamics}

We next measured ${ }^{15} \mathrm{~N}$ spin-lattice/spin-spin relaxation rates as well as heteronuclear NOEs for the mHIV$1-\mathrm{PR}_{1-95}$ at $25^{\circ} \mathrm{C}$, at a field strength of 17.6 Tesla $(750 \mathrm{Mhz})$. These relaxation parameters are sensitive towards motions on the sub-nanosecond timescale. In addition, the $R_{2}$ relaxation rate provides insights into motions on the millisecond to microsecond timescale. Full sets of relaxation data could be extracted for a total of $79\left(5^{\circ} \mathrm{C}\right), 82(4 \mathrm{M}$ urea $), 85(8 \mathrm{M}$ urea $), 87(1 \mathrm{M} \mathrm{GdmCl})$ and $88(25 \%$ acetic acid) residues.

For all five denatured states, the $R_{1}$ values remained more or less constant throughout the sequence, with average values of $1.44 \pm 0.04\left(5^{\circ} \mathrm{C}\right), 1.48 \pm 0.01$ (4 M urea), $1.50 \pm 0.01$ ( $8 \mathrm{M}$ urea), $1.56 \pm 0.03(1 \mathrm{M}$ $\mathrm{GdmCl})$ and $1.36 \pm 0.02 \mathrm{~ms}^{-1}(25 \%$ acetic acid), respectively, Fig. 5. The $\mathrm{N}$ - and the C-termini showed lower $R_{1}$ compared to the rest of the protein, consistent with faster timescale movements usually experienced for chain termini. In all five profiles, we observed a stretch (V77-V82) of significantly lower values followed by a stretch (I84-L89) of significantly increased values. The average $R_{1}$ rates for the acetic acid and for the cold denatured states were clearly reduced compared to those associated with the other two denatured states.

Measurements of the heteronuclear steady-state NOEs showed mostly positive values apart from those associated with the N- and C-terminal regions. The profile of the heteronuclear NOE did not agree with a fully unfolded state, but rather followed a profile of four arcs for all four denatured states.

The $R_{2}$ value is usually the most informative parameter for denatured proteins as it can reveal regions that undergo chemical exchange. For a fully extended protein where chain dynamics is dominated by unrestrained segmental motion, this profile usually adopts the shape of an inverted $U$, with a plateau along the chain and steep drops at the $\mathrm{N}$ - and C-terminal ends ${ }^{62}$. For all five denatured states, the $R_{2}$ profiles deviated from an inverted U-shape. Instead, they displayed a four-arcs-like pattern distributed almost evenly over the sequence, and covering R8-L24, V32-G48, V56-G68 and G78-A95 (cf. Fig. S7 in the SI). This unusual pattern of $R_{2}$ rates persisted at $8 \mathrm{M}$ urea where the unfolded mHIV-1-PR $1-95$ showed a more elongated conformation, as testified by the corresponding $R_{h}$ value (Table 1 ).

The probability function of finding motions at a given angular frequency $\omega$ can be described by the spectral density function $\Theta(\omega)$. As unfolded states cannot be described in terms of an overall rotational correlation time, we instead chose to describe the relaxation data by reduced spectral density mapping ${ }^{63}$. We used the values of $R_{1}, R_{2}$ and the heteronuclear NOE recorded at 17.6 Tesla to derive the spectral density function at three frequencies $\left(0, \omega_{\mathrm{H}}\right.$ and $\omega_{\mathrm{N}}$, cf. Fig. 5). Neither $\Theta\left(\omega_{H}\right)$ nor $\Theta\left(\omega_{N}\right)$ showed large variation in their profiles when plotted against the sequence, in agreement with the related profiles for the hetNOE and the $R_{1}$ values, respectively. Instead, the $J(0)$ values displayed the same pattern of four arcs as described for $R_{2}$ . Of importance we note that the arches mostly revolve around prolines.

\section{Hosted file}

image5.emf available at https://authorea.com/users/420414/articles/526767-the-denaturedstate-of-hiv-1-protease-under-native-conditions

Figure 5: Chain dynamics in HIV-1-PR $1-95$.in denaturant conditions . NMR relaxation rates (a-c) and spectral densities (d-f) in $25 \%$ acetic acid (blue dots), in $8 \mathrm{M}$ urea (red dots), in $4 \mathrm{M}$ urea (orange dots), in $0.9 \mathrm{M} \mathrm{GdmCl}$ (green dots) and in buffer at $5{ }^{\circ} \mathrm{C}$ (black dots). Secondary structures of folded HIV-1-PR $1-95$ are shown in the top.

\section{Experimental validation of molecular dynamics simulation of $D_{0}$}


A replica-exchange simulation of $68 \mu \mathrm{s}$ of $\mathrm{mHIV}-1-\mathrm{PR}_{1-95}$ in water is performed with 110 temperatures in the range from $300 \mathrm{~K}$ to $500 \mathrm{~K}$, as described in the Materials and Methods. The degree of equilibration of the simulation seems acceptable, as testified by the good exchange between replicas (cf. Fig. S8) and by the convergence of the average contact map (cf. Fig. S9).

To validate the simulation, we calculated the average secondary chemical shifts from the simulated trajectory using Sparta $+{ }^{56}$ and compared them with the experimental values extrapolated for $D_{0}$ (Fig. 6). The Pearson's correlation coefficients are $\mathrm{r}=0.68$ for $\mathrm{CA}, \mathrm{r}=0.63$ for $\mathrm{C}^{\prime}, \mathrm{r}=0.67$ for $\mathrm{N}$ and $\mathrm{r}=0.54$ for $\mathrm{HN}$ (also cf. Fig. S10). Thus, the simulated data is in good agreement with the experimental values ( $\mathrm{p}$-value $<10^{-5}$, as calculated from a random bootstrap of the data).

\section{Hosted file}

image6.emf available at https://authorea.com/users/420414/articles/526767-the-denaturedstate-of-hiv-1-protease-under-native-conditions

Figure 6: Secondary chemical shift comparison between experiment and simulation . A comparison between the average secondary chemical shifts of $D_{0}$ predicted by the MD simulation (red line) and those extrapolated from the experiment to zero denaturant (blue line).

The average radius of gyration, $R_{g}$, calculated from the simulated conformations, was $2.19 \pm 0.48 \mathrm{~nm}$. The corresponding $R_{h}$ can be estimated ${ }^{64}$ to be $2.45 \pm 0.61 \mathrm{~nm}$. This is equal, within the error bars, to the hydrodynamic radius $2.51 \pm 0.19 \mathrm{~nm}$ obtained as extrapolation to zero denaturant from the data of Table 1. Another, more qualitative comparison, was done between the experimental and simulated relaxation parameters $R_{1}$ and $R_{2}$. The reason why a direct comparison cannot be done is that a replica-exchange simulation is efficient in sampling the equilibrium conformations of the protein at the price of generating an unphysical time-dependent trajectory, that would be necessary for calculating the NMR relaxation parameters.

To give an approximate estimate of $R_{1}$ from the simulation, we performed 20 plain-MD simulations at fixed temperature $(300 \mathrm{~K})$ starting from 20 conformations extracted from the replica-exchange trajectory. Each simulation lasted for $1 \mathrm{~ns}$, that is the time scale described by the $R_{1}$ parameter. From each simulation we calculated the root mean square fluctuations (RMSF) around the average conformation. We expected that $R_{1}$ is anticorrelated with the RMSF. The comparison between the experimental $R_{1}$ and the (rescaled and shifted) RMSF is displayed in Fig. 7. Although the linear correlation is not high ( $\mathrm{r}=0.21), 74 \%$ of points stay on the same side with respect to the median $\left(\mathrm{p}\right.$-value $\left.=10^{-8}\right)$, suggesting the two curves indicate similar regions of rigid and flexible residues (black bars above the curves).

The values of $R_{2}$ that reflect the conformational freedom of residues on the $\mu$ s-ms timescale, were compared with the total solvent-accessible surface area (SASA) of each amino acid, calculated on the replica-exchange simulation. Again, the linear correlation is low $(\mathrm{r}=0.16)$ but $68 \%$ of the points stay on the same side with respect to the median ( $\mathrm{p}$-value $=10^{-4}$ ), indicating that residues that are experimentally more flexible are those less constrained by other parts of the polymer in the simulation.

\section{Hosted file}

image7.emf available at https://authorea.com/users/420414/articles/526767-the-denaturedstate-of-hiv-1-protease-under-native-conditions

Figure 7: The experimental $R_{1}$ and $R_{2}$ relaxation parameters compared with proxies of the same quantities calculated from the MD simulations. In the case of $R_{1}$, we plotted (in red) the function(2.7-2* $\mathrm{RMSF} / \mathrm{nm}) / \mathrm{ms}$, where the RMSF is calculated from 1ns MD simulations starting from the sampled conformations. In the case of $R_{2}$, we used as proxy the function $\left(8.7-3 * \mathrm{SASA} / n m^{2}\right) / \mathrm{ms}$.

\section{Analysis of the ensemble of conformations of $D_{0}$}

The ensemble of conformations generated by the replica-exchange algorithm at 300K were further analyzed to characterize $D_{0}$. The average $R_{g}$, of value $2.19 \pm 0.48 \mathrm{~nm}$ (cf. Fig. S11), was consistently larger than the 
value $1.28 \mathrm{~nm}$ of the native conformation. The fact that the contact probability between pairs of residues as a function of their distance along the chain is a power law with an exponent [?]-1.8 (cf. Fig. S12) suggests that the chain is, on average, in a coil state.

The average number of contacts is $60.6+-15.3$ (cf. Fig. S13) and the fraction $\mathrm{q}_{\mathrm{N}}$ of native contacts is low ([?] $0.018+-0.011$ ). To be noted the fraction $q_{N}$ of native contacts in the denatured state is poorly correlated with the commonly employed RMSD (cf. Fig. S14). In fact, the RMSD is a highly non-linear function of the diversity between conformations in that it is very sensitive to conformational changes between similar conformations and quite insensitive to large conformational changes between dissimilar conformations. Since the denatured state is expected to be conformationally very heterogeneous, we compared pairs of conformations using the fraction $q$ of common contacts (and we compared a conformation to the native one using the fraction $q_{N}$ of native contacts).

In Fig. 8(a) the distribution of common contacts $q$ between denatured conformations is plotted. Its average is $0.09+-0.08$ but it displays a tail up to 0.7 . Not surprisingly, the denatured state $D_{0}$ thus appears conformationally very heterogeneous. However, its average contact map (cf. Fig. 8(b)) displays welldefined secondary structures that can reach a probability of 0.4 and also tertiary structures populated with probabilities up to 0.15 . Some of these structures are native-like and include the hairpin $\beta 1-\beta 2$, the hairpin $\beta 4-\beta 5$, the hairpin $\beta 5-\beta 6$ and the terminal $\alpha$-helix (cf. Fig. 8(c)). Non-native contacts (cf. Fig. 8(d)) include a set of alternative structures in the region of the hairpin $\beta 4-\beta 5$, some fluctuating structure around P63 and a small amount of tertiary contacts.

\section{Hosted file}

image8.emf available at https://authorea.com/users/420414/articles/526767-the-denaturedstate-of-hiv-1-protease-under-native-conditions

Figure 8: Equilibrium properties of the simulated protein (a) the distribution of similarity $q$ between the conformations of $D_{0},(\mathrm{~b})$ the average contact map of $\mathrm{mHIV}-1-\mathrm{PR}_{1-95}$, (c) the average contact map limited to native contacts, (d) the average contact map limited to non-native contacts

A cluster analysis was performed for the simulated conformations of $D_{0}$ at $300 \mathrm{~K}$ with the Ward algorithm. The fraction $q$ of common contacts was used as underlying metrics for the clustering instead of the more common RMSD because of the reasons described above. We could identify 17 clusters. In Fig. 9, we displayed the three most populated clusters (others can be found in Fig. S15). The most populated cluster (labelled A) has a population of $21 \%$ and is poorly structured; it contains most conformations with a low number of contacts. The only stable structure is a turn involving P63. Clusters B and C have a population of $8 \%$ each. Cluster B displays a non-native $\beta$-hairpin involving residues $39-45$ and the native, C-terminal $\alpha$-helix. Cluster $\mathrm{C}$ displays a $\beta$-hairpin involving residues 56-63, a $\beta$-turn $80-83$ and tertiary contacts between this and the N-terminal region 4-6.

In the other clusters (cf. Fig. S15) particular recurrent non-native contacts in the region 40-50 and the native $\alpha$-helix are seen.

\section{Hosted file}

image9.emf available at https://authorea.com/users/420414/articles/526767-the-denaturedstate-of-hiv-1-protease-under-native-conditions

Figure 9: Cluster analysis of the conformations sampled at 300K . In the dendrogram of structural similarity some clusters are indicated with Latin letters. For the three most populated clusters (labelled A, $\mathrm{B}$ and $\mathrm{C}$ ) the average contact map (normalized to the number of conformations of each cluster, same color code as Fig. 8) and the central conformation (the $\mathrm{N}$ terminus in red, the $\mathrm{C}$ terminus in blue) are shown. The percental equilibrium population is also indicated for each cluster.

\section{Discussion}


The denatured state $D_{0}$ of a protein under native solvent conditions is important to determine its behavior in the cell, but it is usually hard to characterize because of its intrinsic instability and low population. By studying the dependence of NMR observables, like secondary chemical shifts and relaxation parameters, under different denaturing conditions and extrapolating their values to native conditions, we could provide a conformational characterization of $D_{0}$ of the HIV-1-PR $\mathrm{PR}_{1-95}$.

A remarkable result was that the extrapolations of these quantities to native conditions were rather independent on the denaturant and matched the minor population of $D_{0}$ present at native conditions.

In 1976, Pfeil and Privalov showed in a series of experiments ${ }^{9}$ that the unfolding enthalpy of lysozyme, denatured by $\mathrm{pH}, \mathrm{GdmCl}$ and temperature was identical, once the mean energy associated with the denaturant (e.g., the ionization energy in the case of $\mathrm{pH}$ ) was subtracted. From this, they concluded that the states denatured by different means are thermodynamically indistinguishable. Ever since it has been discussed whether the denatured states generated by different means of denaturation were structurally different or not. In the present structural study, the extrapolation of chemical shifts to non-denaturing conditions plays a similar role to that of subtracting the denaturing energy in Privalov's experiment and all the extrapolations seem to agree very well with the presence of a single denatured state.

The interpretation of the raw data produced by NMR experiments in terms of conformational properties of $D_{0}$ is particularly difficult for a state composed of a plethora of heterogeneous conformations. In this case, MD simulations can be a valuable complement to the experimental data because of their ability to probe the system at atomic scale. A critical issue in this respect is whether MD simulations can provide a realistic picture of the state of interest of the protein. To address this concern, we compared the secondary chemical shifts, the hydrodynamic radius and the relaxation parameters predicted by the simulation with the experimental values. The good agreement we found is a consequence of two factors. First, we used advanced sampling techniques of simulation that favor the diffusion in the conformational space of the system, allowing it to sample an heterogeneous conformational space. Second, we employed a force field ${ }^{54}$ that was particularly adjusted to simulate intrinsically-disordered proteins ${ }^{54}$, namely systems with conformational properties that are analogous to those of the denatured state of a structured protein. It is important to stress that the tools to analyze a simulation of the denatured state are different than those typically used for native-like states. For example, while the commonly used RMSD is a poor quantifier of the similarity between pairs of conformations with subtle common features, the fraction $q$ of common contacts being a more sensitive tool.

The detection of transient native and non-native structures in the denatured state of proteins is important to understand their fast folding ${ }^{65,66}$ and their aggregation ${ }^{4,67}$. In the case of a viral protein as HIV-1-PR, such structures can be also relevant as targets of antiviral molecules ${ }^{19-21}$. We found in $D_{0}$ specific secondary structures, both native and non-native, displaying an equilibrium probability of up to [?]30\% and also specific tertiary structures with equilibrium probability of up to [?]10\%. Among them, the most stable elements seem to be the native C-terminal $\alpha$-helix and a non-native $\beta$-like structure at the center of the protein. In particular, our observations transient population of the hairpin $\beta 1-\beta 2$, the hairpin $\beta 4-\beta 5$, the hairpin $\beta 5-\beta 6$ and the terminal $\alpha$-helix. Except for the C-terminal helix, the remaining structures correlate with the arches described by elevated $R_{2}$ values (Fig. 4) and further suggest that the simulations are capturing the details of the ensemble. Furthermore, several substates of $D_{0}$ were identified by cluster analysis, each with peculiar conformational features, both native and non-native. Interestingly, we observed positive secondary chemical shift values for $\mathrm{C}^{\alpha}$ and $\mathrm{C}^{\prime}$ at few places along the chain (Fig. 4), suggesting the presence of transient helicity in regions that in the native structure form $\beta$-strands. Thus, non-native interactions appear relevant to the unfolded state of HIV-1-PR and may play roles in guidance through the folding process.

\section{Conclusions}

The combination of experimental NMR techniques and of advanced MD simulation allowed us to characterize the denatured state of a complex protein as the HIV-1 protease under native conditions. This state displays transient native and non-native secondary and tertiary structures which could be of key relevance for guidance through the complex folding process. The strategy we used for HIV-1 protease can be easily applied to other 
proteins of comparable length.

\section{Acknowledgment}

This work was performed under the auspices of the U.S. Department of Energy by Lawrence Livermore National Laboratory under Contract No. DE- AC52-07NA27344. H.I.R and B.B.K was supported by the Carlsberg Foundation, and B.B.K by the Novo Nordisk Foundation Challenge Program REPIN (\#NNF18OC0033926)

\section{References}

1. Dill KA, Shortle D. Denatured states of proteins. Annu. Rev. Biochem. 1991;60:795-825.

2. Morrone A, McCully ME, Bryan PN, Brunori M, Daggett V, Gianni S, Travaglini-Allocatelli C. The denatured state dictates the topology of two proteins with almost identical sequence but different native structure and function. J. Biol. Chem. 2011;286:3863-3872.

3. Shortle D. The denatured state (the other half of the folding equation) and its role in protein stability. FASEB J. 1996;10:27-34.

4. Fawzi NL, Chubukov V, Clark LA, Brown S, Head-Gordon T. Influence of denatured and intermediate states of folding on protein aggregation. Protein Sci. 2005;14:993-1003.

5. Hetz C, Chevet E, Harding HP. Targeting the unfolded protein response in disease. Nat. Rev. Drug Discov. 2013;12:703-719.

6. Alexandrescu AT, Abeygunawardana C, Shortle D. Structure and Dynamics of a Denatured 131-Residue Fragment of Staphylococcal Nuclease: A Heteronuclear NMR Study. Biochemistry. 1994;33:1063-1072.

7. Reed MAC, Jelinska C, Syson K, Cliff MJ, Splevins A, Alizadeh T, Hounslow AM, Staniforth RA, Clarke AR, Craven CJ, et al. The denatured state under native conditions: a non-native-like collapsed state of N-PGK. J. Mol. Biol. 2006;357:365-372.

8. Mok YK, Kay CM, Kay LE, Forman-Kay J. NOE data demonstrating a compact unfolded state for an SH3 domain under non-denaturing conditions. J. Mol. Biol. 1999;289:619-638.

9. Pfeil W, Privalov PL. Thermodynamic investigations of proteins. Biophys. Chem. 1976;4:23-32.

10. Tanford C, Kawahara K, Lapanje S. Proteins in $6 \mathrm{~m}$ Guanidine Hydrochloride: demonstration of random coil behavior. J. Biol. Chem. 1966;241:1921-1923.

11. Shortle D, Ackerman MS. Persistence of native-like topology in a denatured protein in $8 \mathrm{M}$ urea. Science. 2001;293:487-489.

12. Rösner HI, Poulsen FM. Residue-specific description of non-native transient structures in the ensemble of acid-denatured structures of the all-beta protein c-src SH3. Biochemistry. 2010;49:3246-3253.

13. Sari N, Alexander P, Bryan PN, Orban J. Structure and Dynamics of an Acid-Denatured Protein G Mutant + . Biochemistry. 2000;39:965-977.

14. Teilum K, Thormann T, Caterer NR, Poulsen HI, Jensen PH, Knudsen J, Kragelund BB, Poulsen FM. Different secondary structure elements as scaffolds for protein folding transition states of two homologous four-helix bundles. Proteins Struct. Funct. Genet. 2005;59:80-90.

15. Wang Y, Shortle D. Residual helical and turn structure in the denatured state of staphylococcal nuclease: analysis of peptide fragments. Fold. Des. 1997;2:93-100.

16. Ackerman MS, Shortle D. Robustness of the long-range structure in denatured staphylococcal nuclease to changes in amino acid sequence. Biochemistry. 2002;41:13791-13797. 
17. Bruun SW, Iesmantavicius V, Danielsson J, Poulsen FM. Cooperative formation of native-like tertiary contacts in the ensemble of unfolded states of a four-helix protein. Proc. Natl. Acad. Sci. U. S. A. 2010;107:13306-13311.

18. Ishima R, Torchia DA, Lynch SM, Gronenborn AM, Louis JM. Solution structure of the mature HIV-1 protease monomer: insight into the tertiary fold and stability of a precursor. J. Biol. Chem. 2003;278:4331143319.

19. Broglia RA, Provasi D, Vasile F, Ottolina G, Longhi R, Tiana G. A folding inhibitor of the HIV-1 protease. Proteins Struct. Funct. Genet. 2005;62:928-933.

20. Broglia RA, Levy Y, Tiana G. HIV-1 protease folding and the design of drugs which do not create resistance. 2008;18:60-66.

21. Kimura S, Broglia RA, Tiana G. Thermodynamics of strongly allosteric inhibition: a model study of HIV-1 protease. Eur. Biophys. J. 2012 Oct 8:1-13.

22. Plaxco KW, Simons KT, Baker D. Contact order, transition state placement and the refolding rates of single domain proteins. J. Mol. Biol. 1998;277:985-994.

23. Kuzmic P. Kinetic assay for HIV proteinase subunit dissociation. Biochem. Biophys. Res. Commun. 1993;191:998-1003.

24. Noel AF, Bilsel O, Kundu A, Wu Y, Zitzewitz JA, Matthews CR. The folding free-energy surface of HIV-1 protease: insights into the thermodynamic basis for resistance to inhibitors. J. Mol. Biol. 2009;387:10021016.

25. Ishima R, Ghirlando R, Tozser J, Gronenborn AM, Torchia DA, Louis JM. Folded monomer of HIV-1 protease. J. Biol. Chem. 2001;276:49110-49116.

26. Caldarini M, Sonar P, Valpapuram I, Tavella D, Volonte C, Pandini V, Vanoni MA, Aliverti A, Broglia RA, Tiana G, et al. The complex folding behavior of HIV-1-protease monomer revealed by optical-tweezer single-molecule experiments and molecular dynamics simulations. Biophys. Chem. 2014;195C:32-42.

27. Rosner HI, Caldarini M, Prestel A, Vanoni MA, Broglia RA, Aliverti A, Tiana G, Kragelund BB. Cold Denaturation of the HIV-1 Protease Monomer. Biochemistry. 2017;56:1029-1032.

28. Tomasselli AG, Heinrikson RL. Targeting the HIV-protease in AIDS therapy: a current clinical perspective. Biochim. Biophys. Acta. 2000;1477:189-214.

29. Cecconi F, Micheletti C, Carloni P, Maritan A. Molecular dynamics studies on HIV-1 protease drug resistance and folding pathways. Proteins Struct. Funct. Genet. 2001;43:365-372.

30. Kimura S, Caldarini M, Broglia RA, Dokholyan N V, Tiana G. The maturation of HIV-1 protease precursor studied by discrete molecular dynamics. Proteins Struct. Funct. Genet. 2014;82:633-639.

31. Bhavesh NS, Panchal SC, Mittal R, Hosur R V. NMR identification of local structural preferences in HIV-1 protease tethered heterodimer in 6 M guanidine hydrochloride. FEBS Lett. 2001;509:218-224.

32. Bhavesh NS, Sinha R, Mohan PMK, Hosur R V. NMR elucidation of early folding hierarchy in HIV-1 protease. J. Biol. Chem. 2003;278:19980-19985.

33. Rout MK, Hosur R V. Fluctuating partially native-like topologies in the acid denatured ensemble of autolysis resistant HIV-1 protease. Arch. Biochem. Biophys. 2009;482:33-41.

34. Chatterjee A, Mridula P, Mishra RK, Mittal R, Hosur R V. Folding regulates autoprocessing of HIV-1 protease precursor. J. Biol. Chem. 2005;280:11369-11378.

35. Kay L, Keifer P, Saarinen T. Pure absorption gradient enhanced heteronuclear single quantum correlation spectroscopy with improved sensitivity. J. Am. Chem. Soc. 1992;114:10663-10665. 
36. Kay LE, Ikura M, Tschudin R, Bax A. Three-dimensional triple-resonance NMR spectroscopy of isotopically enriched proteins. J. Magn. Reson. 1990;89:496-514.

37. Clubb RT, Thanabal V, G. Wagner. A constant-time three-dimensional triple-resonance pulse scheme to correlate intraresidue $1 \mathrm{HN}, 15 \mathrm{~N}$, and 13C' chemical shifts in 15N-13C-labelled proteins. J. Magn. Reson. 1992;97:213-217.

38. Bax A, Ikura M. An efficient 3D NMR technique for correlating the proton and $15 \mathrm{~N}$ backbone amide resonances with the alpha-carbon of the preceding residue in uniformly $15 \mathrm{~N} / 13 \mathrm{C}$ enriched proteins. J. Biomol. NMR. 1991;1:99-104.

39. Wittekind M, L. Mueller. HNCACB, a high-sensitivity 3D NMR experiment to correlate amide-proton and nitrogen resonances with the alpha- and beta-carbon resonances in proteins. J. Magn. Reson. Ser. B. 1993;101:201-205.

40. Grzesiek S, Bax A. Correlating backbone amide and side chain resonances in larger proteins by multiple relayed triple resonance NMR. J. Am. Chem. Soc. 1992;114:6291-6293.

41. Panchal SC, Bhavesh NS, V. Hosur R. Improved 3D triple resonance experiments, HNN and HN(C)N, for $\mathrm{HN}$ and $15 \mathrm{~N}$ sequential correlations in $(13 \mathrm{C}, 15 \mathrm{~N})$ labeled proteins: application to unfolded proteins. J. Biol. Chem. 2001;20:135-147.

42. Zhang O, Kay LE, Olivier JP, Forman-Kay JD. Backbone $1 \mathrm{H}$ and $15 \mathrm{~N}$ resonance assignments of the N-terminal SH3 domain of drk in folded and unfolded states using enhanced-sensitivity pulsed field gradient NMR techniques. J. Biomol. NMR. 1994;4:845-858.

43. L. E. Kay, Torchia DA, Bax A. Backbone dynamics of proteins studied by $15 \mathrm{~N}$ inverse-detected heteronuclar NMR spectroscopy: Application to staphylococcal nuclease. Biochemistry. 1989;28:8972-8979.

44. Balayssac S, Delsuc M-A, Gilard V, Prigent Y, Malet-Martino M. Two-dimensional DOSY experiment with Excitation Sculpting water suppression for the analysis of natural and biological media. J. Magn. Reson. 2009;196:78-83.

45. Wishart DS, Bigam CG, Yao J, Abildgaard F, Jane HD, Oldfield E, Markley JL, Sykes BD. Chemical shift referencing in biomolecular NMR. J. Biomol. NMR. 1995;6:135-140.

46. Delaglio F, Grzesiek S, Vuister GW, Zhu G, Pfeifer J, Bax a. NMRPipe: a multidimensional spectral processing system based on UNIX pipes. J. Biomol. NMR. 1995;6:277-93.

47. Orekhov VY, Jaravine VA. Analysis of non-uniformly sampled spectra with multi-dimensional decomposition. Prog. Nucl. Magn. Reson. Spectrosc. 2011;59:271-92.

48. Vranken WF, Boucher W, Stevens TJ, Fogh RH, Pajon A, Llinas M, Ulrich EL, Markley JL, Ionides J, Laue ED. The CCPN data model for NMR spectroscopy: development of a software pipeline. Proteins. 2005;59:687-96.

49. D'Auvergne EJ, Gooley PR. Optimisation of NMR dynamic models I. Minimisation algorithms and their performance within the model-free and Brownian rotational diffusion spaces. J. Biomol. NMR. 2009;40:107119.

50. D'Auvergne EJ, Gooley PR. Optimisation of NMR dynamic models II. A new methodology for the dual optimisation of the model-free parameters and the Brownian rotational diffusion tensor. J. Biomol. NMR. 2009;40:121-133.

51. Nilsson M. The DOSY Toolbox: a new tool for processing PFG NMR diffusion data. J. Magn. Reson. 2009;200:296-302.

52. MATLAB, The MathWorks, Inc. 
53. Klein-Seetharaman J, Oikawa M, Grimshaw SB, Wirmer J, Duchardt E, Ueda T, Imoto T, Smith LJ, Dobson CM, Schwalbe H. Long-range interactions within a nonnative protein. Science. 2002;295:1719-1722.

54. Robustelli P, Piana S, Shaw DE. Developing a molecular dynamics force field for both folded and disordered protein states. Proc. Natl. Acad. Sci. U. S. A. 2018;115:E4758-E4766.

55. Pall S, Zhmurov A, Bauer P, Abraham M, Lundborg M, Gray A, Hess B, Lindahl E. Heterogeneous parallelization and acceleration of molecular dynamics simulations in GROMACS. J. Chem. Phys. 2020;153:134110.

56. Shen Y, Bax A. SPARTA+: a modest improvement in empirical NMR chemical shift prediction by means of an artificial neural network. J. Biomol. NMR. 2010;48:13-22.

57. Dagil R, Knudsen MJ, Olsen JG, O'Shea C, Franzmann M, Goffin V, Teilum K, Breinholt J, Kragelund BB. The WSXWS Motif in Cytokine Receptors Is a Molecular Switch Involved in Receptor Activation: Insight from Structures of the Prolactin Receptor. 2012;20:270-282.

58. Kogo H, Takeuchi K, Inoue H, Kihara H, Kojima M, Takahashi K. Urea-dependent unfolding of HIV1 protease studied by circular dichroism and small-angle X-ray scattering. Biochim. Biophys. Acta. 2009;1794:70-74.

59. Todd MJ, Semo N, Freire E. The structural stability of the HIV-1 protease. J. Mol. Biol. 1998;283:475488.

60. Levy Y, Caflisch A, Onuchic JN, Wolynes PG. The folding and dimerization of HIV-1 protease: evidence for a stable monomer from simulations. J. Mol. Biol. 2004;340:67-79.

61. Louis JM, Ishima R, Aniana A, Sayer JM. Revealing the dimer dissociation and existence of a folded monomer of the mature HIV-2 protease. Protein Sci. 2009;18:2442-53.

62. Schwalbe H, Fiebig KM, Buck M, Jones JA, Grimshaw SB, Spencer A, Glaser SJ, Smith LJ, Dobson CM. Structural and dynamical properties of a denatured protein. Heteronuclear 3D NMR experiments and theoretical simulations of lysozyme in 8 M urea. 1997;36:8977-8991.

63. Farrow N, Zhang O, Szabo A, Torchia D, Kay LE. Spectral density function mapping using $15 \mathrm{~N}$ relaxation data exclusively. J. Biomol. NMR. 1995;6:153-62.

64. Nygaard M, Kragelund BB, Papaleo E, Lindorff-Larsen K. An Efficient Method for Estimating the Hydrodynamic Radius of Disordered Protein Conformations. Biophys. J. 2017;113:550-557.

65. Karplus M, Weaver DL. Protein-folding dynamics. Nature. 1976;260:404-406.

66. Broglia R, Tiana G. Hierarchy of events in the folding of model proteins. J. Chem. Phys. 2001;114:72677273.

67. Broglia RA, Tiana G, Pasquali S, Roman HE, Vigezzi E. Folding and aggregation of designed proteins. Proc. Natl. Acad. Sci. U. S. A. 1998;95:12930-12933. 